\title{
Nager syndrome
}

INSERM

\section{Source}

INSERM. (1999). Orphanet: an online rare disease and orphan drug data base. Nager syndrome. ORPHA:245

Nager syndrome, also called Nager acrofacial dysostosis (NAFD) is a congenital malformation syndrome characterized by mandibulofacial dystosis (malar hypoplasia, micrognathia, external ear malformations) and variable preaxial limb defects. 